\title{
Balancing dual roles in end-of-life research
}

By Wanda Martin, Meredith Grey, Terry Webber, Linnea Robinson, Nancy Hartt, Moira Cairns and Kelli Stajduhar

\section{Abstract}

Ethical and practical issues are sure to arise from the majority of research studies done with palliative populations. Whether it is feeling opportunistic, being emotionally available, or struggling with witnessing a gap in service and needs of the participants receiving care, nurses involved in research find a way to balance both roles to meet the needs of the participants as well as the study (McIlfatrick, Sullivan, \& McKenna, 2006). This paper highlights some of the practical and ethical issues that arise when frontline nurses also take on the role of research assistant for studies with palliative populations. Specifically, the authors highlight their personal experiences based on their research assistant work on a study examining family caregiver coping in end-of-life cancer care. The authors discuss the "balancing act" of taking on these dual roles and offer recommendations on how to be with and approach people when doing research at the end of life using a framework based on Swanson's Theory of Caring (1991).

Nursing is a wide-ranging profession offering the opportunity for a variety of roles in a multitude of settings. In 2005 , nearly $75 \%$ of Canadian nurses were employed in direct patient care and $0.7 \%$ in research as their primary area of responsibility (Canadian Institute for Health Information [CIHI], 2006). Nurses involved in direct patient care have a particular knowledge and way of being with patients and families that makes them a great asset for researchers in assisting with data collection. However, involvement in research places these nurses in a position of having two roles - that of frontline practising nurse and that of nurse involved in doing research (Colbourne \& Sque, 2004). Concerns often arise around the sometimes conflicting obligations of balancing the sensitivities and vulnerabilities of participants, while at the same time attempting to adhere to issues of

Wanda Martin, RN, MN, Project Coordinator, Centre on Aging, University of Victoria, Sedgewick Building, Room A104, 3800

Finnerty Road, Victoria, B.C. V8P 5 C2

E-mail:wmartin@uvic.ca

Meredith Grey, RN, BSN, Quality Improvement Consultant for Heart Health and Adult ICU, Quality and Patient Safety, Vancouver Island Health Authority, Victoria, B.C.

Terry Webber, RN, Research Assistant, University of Victoria, Clinical Resource Nurse, Hospice Palliative Care Consult Team, Surrey Memorial Hospital, Surrey, B.C.

Linnea Robinson, RN, BSN, Research Assistant, University of Victoria, Home Health Nurse (LTC) Fraser Health Authority, White Rock, B.C.

Nancy Hartt, RN, BSN, Research Assistant, University of Victoria, Clinical Trials Nurse and Oncology Nurse, BC Cancer Agency-Centre for the Southern Interior, Kelowna, B.C.

Moira Cairns, BA, RSW, Research Assistant, University of Victoria, Victoria, B.C.

Kelli I. Stajduhar, RN, PhD, Assistant Professor, School of Nursing and Centre on Aging, University of Victoria, Victoria, B.C. study rigor (Daly \& Rosenfeld, 2003). This paper highlights some of the ethical and practical issues that arise when nurses take on the dual roles of clinician and researcher, especially with end-of-life populations. The authors discuss this "balancing act" and offer recommendations on how to approach and be with people when doing research at the end of life. Swanson's middle range theory of caring (1991) provides a framework for the discussions.

\section{Setting the context}

The authors of this paper were brought together when they were hired to work on a multi-site research project funded by the Canadian Cancer Society entitled, "Family Caregiver Coping in End-of-Life Cancer Care." The project was led by a nurse researcher (K.S.), coordinated by a Master's prepared nurse (W.M.) and comprised several research assistants, five of whom were registered nurses (RNs) and who were responsible for conducting in-depth semistructured interviews and administering a structured questionnaire to a sample of family caregivers of people who were dying of cancer. The primary purpose of the study was concerned with the question of why some caregivers seem to cope better than others while under similar caregiving demands. The research assistants were not directly involved with the care of the dying family member, but were working in home nursing care or cancer care, having experience and expertise with the population being studied. The research participants were recruited through hospice, homecare, and pain and symptom management clinics in three urban areas in British Columbia. Qualitative interviews were done with 28 current caregivers and 17 bereaved caregivers. More than 300 family caregivers participated in the quantitative phase of the study. In order to prepare the research assistants, many of whom had little formal research training, two fullday training sessions were led by the principal investigator. The training covered topics such as study design, interviewing skills, research ethics and the pragmatics of being involved in a research project.

As the study is nearing completion, results are not yet available. However, in the process of interviewing family caregivers for the study, a number of practical and ethical issues arose, resulting from the dual roles the research assistants held. Research team members (the principal investigator, project coordinator and research assistants) thought it would be interesting to explore these dual roles and what they were like for the research assistants. While this was not conceived as a research study, the team believed that the lessons learned from their collective experiences might benefit other oncology and palliative care nurses who also took on research roles. The project coordinator began recording issues that arose in relation to these dual roles and four of the research assistants provided their written thoughts and feelings of their experience of working on the research project to the project coordinator. This information formed the basis for this paper. As a way to conceptualize the information, the authors used Swanson's middle range theory of caring (1991) as a framework to describe the dual roles of nurse and researcher. This theory is based on knowing, being with, doing for, enabling, and maintaining belief. As a nursing theory, this is easily demonstrated in clinical nursing practice. However, from a research perspective, it is less obvious how this theory applies, as there are potential conflicts in clinical practice while actively collecting data for a research project.

\section{Knowing}

According to Swanson (1991), "knowing" is approaching a situation with no assumptions but, rather, centring on the one cared for and assessing their experience to try to understand their reality. 
Knowing from a nursing perspective was close to being an instinctual act for some of the nurses on this project who have worked for a number of years in direct patient care. Knowing from a research perspective was a less familiar experience.

The approach taken in the family caregiver coping study was to be centred on the participants, not only to understand their realities as a part of the purpose and goal of the study, but also to respect them as individuals. This approach corresponds with the nurses' "knowing", however, there were occasions when the research assistants were faced with making choices between their nurse and researcher roles. For example, the research assistants oftentimes found themselves in conflict between being a healer or giver-of-care and feeling opportunistic. The research assistants sometimes felt they were taking advantage of the family of the dying individual to fulfill a research agenda. These feelings tended to surface more often while doing quantitative interviews, which were structured and left less time for the research participant to delve into and fully express their experiences.

Conducting in-depth qualitative interviews was more satisfying for some of the research assistants as it offered the opportunity to explore the participants' experiences and realities and how they were coping with the demands of care. This approach allowed participants to more fully tell their stories. It was apparent in many interactions that being interviewed offered participants the opportunity of being listened to and to gain a sense of their own ability to cope with and learn from their experience. As already implied, conducting the quantitative interviews tended to be less satisfying as there were fewer opportunities for back-and-forth conversational exchanges that are more akin to qualitative interviewing. While quantitative interviewing does not preclude the caring approach, the authors' experience was that it can sometimes restrict the quality of the exchange with the participant.

In order to avoid feeling opportunistic, it was key for the research assistants to recognize their presence as a listener as being beneficial to the participant, whether it be with qualitative or quantitative interviews. When approaching research from a theory of caring, research assistants were less likely to exploit the participant relationship for the sake of the research agenda (Head \& Ritchie, 2004). Maintaining the research standards of obtaining informed consent and respecting participants' decisions on the amount of participation they were able and willing to do supported both the participant and the research assistant in preventing the negative feeling derived from opportunism.

Doing research in the area of end-of-life, however, is fraught with ethical concerns. Researchers and their team members need to remember that many participants are often altruistic and find meaning in contributing to knowledge that may help others (Head \& Ritchie, 2004). Therefore, be it qualitative interviews that allow for more in-depth conversations, or quantitative interviews consisting of direct questions, the participants' involvement can be a valuable part of their process. Knowing ethical standards for research and respecting the participants' decisions on their level of involvement will help nurse researchers and research assistants to balance their dual roles.

\section{Being with}

The second caring process, "being with", is being emotionally open, conveying that the person's experience matters, and sharing feelings while not burdening (Swanson, 1991). This ability to be empathetic is a valued trait in nursing (Kunyk \& Olson, 2001). It is not enough to be physically present with the person. The research assistants reported their need to understand the realities of the participant from the research perspective while being emotionally present. Research assistants wanted to balance the analytical scientific needs of the study with the empathy needed to be a caring nurse.
The title "nurse" can have a strong influence on the research process. It is the cultural identity of nurses, as empathetic practitioners who are accustomed to dealing with private matters, which gives people permission to discuss social taboos and encourages disclosure (Leslie \& McAllister, 2002). This willingness of participants to be involved in a study and to disclose to research assistants opens doors that may not be available to non-nurses. However, there is a delicate balance in having this advantage and there are pitfalls that need to be guarded against.

There can be a sense of indebtedness or dependence on the nurse, which contributes to the participation in the study at the risk of involuntary consent (Head \& Ritchie, 2004). Research staff needs adequate training and education to recognize and avoid such situations. Clear role delineation is important for both the researcher and participant to ensure voluntary consent (Clarke \& Johnson, 2003).

It is also important to be aware of the power of the default of being a nurse and responding to the participant from a teaching or counselling perspective with a focus on intervention instead of the research perspective of data collection. The cultural identity of nurse is projected from others as well as being internalized in the nurse researcher (MacIntosh, 2003).

Being emotionally open may lead to subjective bias, which may cloud the interview, with the nurse researcher or research assistant losing sight of the research objectives (Field, 1991). It is important, as researchers, to identify our values and beliefs, to acknowledge subjectivity, and to continue with self-evaluation throughout the data collection period and analysis process (Polit \& Hungler, 1999). However, the tension remains between research being used as therapy and research being utilized purely for data collection. "Being with" includes sharing feelings, and this use of therapeutic communication can be an asset employed to alleviating suffering of the participant (Leslie \& McAllister, 2002). One research assistant documented her experience of "being with" the participant in the interview:

One caregiver was extremely distraught when I visited her. Her husband's condition was changing rapidly after a fall, marking the end of his ability to mobilize on his own. While he sat in the next room, frequently calling out to her, the caregiver showed signs of her inability to stay focused on the interview for long, due, in part, to his calling out and due to her own stresses that she recited in her belief that she was losing him. Despite a reassurance that she could stop the interview at any time, she insisted that, while she was not sure if her info was helpful to hear, it was helpful for her to share. She stated, "My husband and I are so different in times like this... when he is stressed, he keeps to himself, but me, I have to talk!" I was amazed that despite a turbulent time, this caregiver's coping style continued to need a place to share openly and verbally of what was going on for her.

\section{Doing for and enabling}

The third and fourth components of Swanson's (1991) theory of nursing care are the outward physical manifestation of the act of caring: the doing and enabling. "Doing for" involves doing for another what he or she would do for him/herself if it were possible. With the associated actions of comforting, anticipating, protecting, and performing skilfully, it is perhaps the most easily recognizable of the aspects of nursing care (Swanson). "Enabling" is facilitating another through life transitions and unfamiliar events by using expert knowledge. It involves providing information and offering emotional support while validating the other's feelings (Swanson). While two separate categories in Swanson's theory, "doing for" and "enabling" are grouped together here because the authors feel that, in the context of this research, what they are doing as research assistants is enabling caregivers through the life transition of losing a family member. 
Often, as a result of the propensity for nurses to "do" care, those who are working within the field of research become torn between the familiar role of being the caring, empathetic and pro-active doer, and the need to collect unbiased and accurate data (Asselin, 2003). This tension is highlighted when a nurse involved in research finds him/herself recognizing that the participant has an actual need requiring attention, yet they are constrained by the limits of the researcher role.

The commonness of this situation makes it vital that nurses, new to the realm of research, are prepared to encounter such tension. When faced with role confusion, research assistants benefit by keeping notes on their thoughts, feelings and responses to observations and interviews (Asselin, 2003). It is vital that research assistants have a strong support system. Team education on ethical conflict resolution and negotiation skills can help the nurse research assistants cope with role tension when it occurs, with peer debriefing and discussions, giving research assistants a place to seek advice and resolve role conflict (Beale \& Wilkes, 2001). Finally, research teams work best when headed by someone with experience who can lead from a position of having knowledge of this emotional pull to "do care", and can offer pertinent guidance and support (Mitchell \& Jones, 2004). This leadership is vital in supporting novice research assistants who are also nurses.

When research assistants are confronted with the unsettling feeling that a research participant is not receiving the care they should, there are three possible actions: never break confidentiality and keep concerns to themselves, break confidentiality and discuss the situation with a colleague in an effort to address the needs of the participant, or recommend to the participant that they contact their care provider and address this issue (Casarett \& Karlawish, 2000; Field, 1991). Of these three choices, the final choice is the only one that attends to both the needs of the participant, and the research priorities. However, if nurses involved in research intend to intervene in a care situation, such efforts should preferably be made after data collection, if this is ethically viable. With the exception of situations that require prompt intervention, research assistants should try to avoid the temptation to intervene during the interview and, instead, answer questions or make referrals at the end of the session (Colbourne \& Sque, 2004). Of course, personal judgment will determine the best interest of the participant and termination of the interview may be necessary to attend to the ethical obligations within the bounds of research.

When it comes to the emotional component of caring for the participant, it has been the experience of the research assistants that the need for validation of the participant's feelings should be attended to immediately. These authors contend that if you do not address some piece of the participant's emotional needs, you risk the participant feeling isolated. The participant may also feel they have risked sharing a very intimate piece of their experience with no consideration of their feelings, which may jeopardize open dialogue. However, if the research assistant acknowledges feelings in a way that overemphasizes the situation, there is a concern that the participant will focus on positive details and, therefore, skew the research. It is a delicate balance, demanding the use of critical and instinctive judgments to know if just enough emotional support is given at just the right time. Information support can wait until the interview is over, but emotional support often requires immediate attention. The "doing for" can be a complicated aspect of research work in balancing the needs of the participant with those of the study.

\section{Maintaining belief}

The final category in Swanson's (1991) theory is maintaining belief. "Maintaining belief" involves having a hope-filled attitude, offering realistic optimism while assisting others to attain, maintain or regain meaning in their experience (Swanson).

During the course of this study, some of the research assistants reported finding themselves in conflict between their role as one who enables the life transition of a family member dying, and the response by some participants to the title of the study. The title "Family Caregiver Coping in End-of-life Cancer Care" was on every piece of study information sent to the participants. As with other studies, the research team found that the term "end of life" could be emotionally laden for those who found themselves in such a situation (AddingtonHall, 2002). Caregivers who were participants in the study reported feeling a loss of hope on reading these words. Although all participants were approached through a hospice, palliative care program or pain and symptom management clinic and were aware of the seriousness of the illness of their family member, seeing the words on paper evoked strong reaction in some. The research assistants were then faced with taking action as nurses to enable the caregivers in maintaining belief by suggesting the term "end of life" be removed from the study documents. Their advocacy role was part of the doing and enabling of their nursing practice. By being both nurse and research assistant, the study was enhanced by the nurses' understanding of the importance of maintaining belief within this population. When we changed the study documents, accrual increased by $37.5 \%$.

\section{Implications}

Ethical and practical issues are sure to arise from the majority of research studies done with palliative populations (Addington-Hall, 2002). The experiences of and lessons learned from being involved in this project demonstrate that it is possible to balance the roles of being a nurse and being involved in research. With leadership from an experienced nurse researcher, adequate training, and the opportunity to continually debrief situations when working with sensitive and vulnerable populations, nurses can and do provide important insights into improving research processes by ensuring that ethical standards are not only applied to the research design itself, but also to the participants involved in research. Hiding the nurse within the researcher may go against the philosophy of nursing (Colbourne \& Sque, 2004) and distract from the satisfaction of doing nursing research. There are ways to balance these dual roles. Recognizing our biases by recording our thoughts and continuing with self-evaluation are tools that nurses benefit from in clinical practice as well as in research. It may be beneficial to have research assistants who work clinically keep a diary focusing specifically on balancing dual roles of researcher and nurse. Recognizing conflicting demands for each role as they occur may be the best way to resolve the issues (Colbourne \& Sque). Research assistants, working in especially sensitive areas, such as oncology and palliative care. can also benefit from skilled preparation and training with ongoing supervision and support to be better equipped to engage with and resolve difficult issues as they arise (Johnson \& Clarke, 2003). Future research in the area of balancing dual roles will help to recognize and maximize the skills of nurses who work clinically to engage in and enhance nursing research. 


\section{References}

Addington-Hall, J. (2002). Research sensitivities to palliative care patients. European Journal of Cancer Care, 11, 220-224.

Asselin, M.E. (2003). Insider research. Issues to consider when doing qualitative research in your own setting. Journal for Nurses in Staff Development, 19(2), 99-103.

Beale, B., \& Wilkes, L. (2001). Nurse researcher: Always a researcher, sometimes a nurse. Collegian, 8(4), 33-39.

Canadian Institute for Health Information. (2006). Workforce trends of registered nurses in Canada, 2005. Retrieved March 5, 2007, from http://secure.cihi.ca/cihiweb/dispPage.jsp?cw_page=PG_590_E\& cw_topic $=590 \&$ cw_rel=AR_20_E

Casarett, D.J., \& Karlawish, J.H.T. (2000). Are special ethical guidelines needed for palliative care research? Journal of Pain and Symptom Management, 20(2), 130-139.

Clarke, J.M., \& Johnson, B. (2003). Collecting sensitive data: The impact on researchers. Qualitative Health Research,13(3), 421434.

Colbourne, L., \& Sque, M. (2004). Split personalities: Role conflict between the nurse and the nurse researcher. Journal of Research in Nursing, 9(4), 297-304

Daly, B.J., \& Rosenfeld, K. (2003). Maximizing benefits and minimizing risks in health services research near the end of life. Journal of Pain and Symptom Management, 25(4), S33-S42.

Field, P.A. (1991). Doing fieldwork in your own culture. In J.M. Morse (Ed.), Qualitative Nursing Research: A Contemporary Dialogue (Revised). California: Sage Publications.
Head, B., \& Ritchie, C. (2004). Researching end-of-life care: Challenges, strategies and opportunities. Home Healthcare Nurse, 22(1), 37-44.

Johnson, B., \& Clarke, J.M. (2003). Collecting sensitive data: The impact on researchers. Qualitative Health Research, 13(3), 421434.

Kunyk D., \& Olson, J.K. (2001). Clarification of conceptualizations of empathy. Journal of Advanced Nursing, 35(3), 317-325.

Leslie, H., \& McAllister, M. (2002). The benefits of being a nurse in critical social research practice. Qualitative Health Research, 12(5), 700-712.

MacIntosh, J. (2003). Reworking professional nursing identity. Western Journal of Nursing Research, 2(6), 725-741.

McIlfatrick, S., Sullivan, K., \& McKenna, H. (2006). Exploring the ethical issues of the research interview in the cancer context. European Journal of Oncology Nursing, 10, 39-47.

Mitchell, T., \& Jones, S. (2004). Leading and co-ordinating a multinurse researcher project. Nurse Researcher, 12(2), 42-55.

Polit, D.F., \& Hungler, B.P. (1999). Nursing research principles and methods (6th ed.). Philadelphia: Lippincott Williams \& Wilkins.

Swanson, K.M. (1991). Empirical development of a middle range theory of caring. Nursing Research, 40(3), 161-6. 